\title{
Precision of Phoneme Boundaries Derived using Hidden Markov Models
}

\author{
Ladan Baghai-Ravary, Greg Kochanski, John Coleman \\ Phonetics Laboratory, Oxford University \\ ladan.baghai-ravary@phon.ox.ac.uk
}

\begin{abstract}
This paper will be presented at Interspeech 2009, the $10^{\text {th }}$ Annual Conference of the International Speech Communication Association, Brighton, UK, 6-10 September 2009, under the sponsorship of the International Speech Communication Association (ISCA). It may be found at http://kochanski.org/gpk/papers/2009/200906boundaries.pdf .
\end{abstract}

\begin{abstract}
Some phoneme boundaries correspond to abrupt changes in the acoustic signal. Others are less clear-cut because the transition from one phoneme to the next is gradual.

This paper compares the phoneme boundaries identified by a large number of different alignment systems, using different signal representations and Hidden Markov Model structures. The variability of the different boundaries is analysed statistically, with the boundaries grouped in terms of the broad phonetic classes of the respective phonemes.

The mutual consistency between the boundaries from the various systems is analysed to identify which classes of phoneme boundary can be identified reliably by an automatic labelling system, and which are ill-defined and ambiguous.

The results presented here provide a starting point for future development of techniques for objective comparisons between systems without giving undue weight to variations in those phoneme boundaries which are inherently ambiguous. Such techniques should improve the efficiency with which new alignment and HMM training algorithms can be developed.
\end{abstract}

\section{Introduction}

Almost all current acoustic-phonetic speech technology (speech recognition, understanding, alignment and synthesis systems) is based on the concept of speech as a sequence of discrete speech units (phonemes). However in practice most realisations of these units are far from discrete - they blend into one another, so that any sharply defined boundaries are arbitrary to some degree.

In the development of a new speech system, one of the first steps generally involves analysing a large database to identify the locations of individual phonemes or phoneme groups. The database being analysed is often prohibitively large for manual labelling, so an automatic system is required, but the design of such an automatic system is problematic because it is not clear how to assess its performance.

The most common method of assessing labelling accuracy is to compare the automatic system's labels with a 'goldstandard' set defined by one or more phoneticians $[1,2]$, but this is inherently subjective and requires much manual effort To avoid these difficulties, we have developed an objective measure of the quality of the alignment system, that operates without reference to human interpretations. This measure responds to inconsistencies (where the time difference between the different systems' labels is unpredictable), and it is not affected by consistent differences between systems (where one system places a particular label earlier or later than another).

Both manual and automatic segmentation accuracy varies as a function of the phonemes on either side of the boundary [3]; some boundaries are accurately predictable from one system to another, while others vary greatly and are essentially unpredictable. We focus on the phoneme-dependency of accuracy, measured by system-to-system predictability.

The approach described here bears some similarity to that of Kominek and Black [4], but we do not average the results of alignment systems to obtain a definitive set of labels - we compares every individual alignment system's result with every other one and assesses how precisely the labels can be located - not where they should be. It allows for the fact that some alignment systems may tend to label a particular type of transition early or late relative to another. Such consistent discrepancies do not indicate a significant difference in precision between alignment systems, and so do not affect the results of this analysis.

\section{Method}

Our approach is to use a cohort of results from automatic systems to estimate the variability of individual labels. By building many different phonetic alignment systems with diverse characteristics and comparing the individual labels between these systems, it is possible to determine which boundaries are ambiguous and which can be defined accurately, without reference to human labels.

\subsection{Phoneme Groups}

There are very many possible phoneme-to-phoneme boundaries (typically 2000 or more, depending on the details of the phonetic inventory). This is too many for simple interpretation of any analysis based on individual phoneme identities.

Furthermore, many of these phoneme-pairs are very rare in natural speech, and any statistics derived from such small numbers of examples would be unreliable. To avoid these problems, phonemes can be grouped into broad phonetic classes before calculating any statistics (see Table 1, which also shows the abbreviations for each class, as used in the rest of the paper).

Table 1. Broad phonetic classes (SAMPA [5]).

\begin{tabular}{|c|c|c|}
\hline Class & Abbr. & Phones (SAMPA) \\
\hline Nasal & Nas & $\mathrm{m}, \mathrm{n}, \mathrm{N}$ \\
Plosive & Plo & $\mathrm{b}, \mathrm{d}, \mathrm{g}, \mathrm{k}, \mathrm{p}, \mathrm{t}$ \\
Affricate & Aff & $\mathrm{dZ}, \mathrm{tS}$ \\
Fricative & Frc & $\mathrm{D}, \mathrm{S}, \mathrm{T}, \mathrm{Z}, \mathrm{f}, \mathrm{h}, \mathrm{s}, \mathrm{v}, \mathrm{z}$ \\
Vowel & Vow & $@, \mathrm{~A}, \mathrm{E}, \mathrm{I}, \mathrm{O}, \mathrm{U}, \mathrm{V},\{, 3, \mathrm{i}, \mathrm{u}, \mathrm{Q}$ \\
Approximant & App & $\mathrm{r}, \mathrm{j}, \mathrm{l}, \mathrm{w}$ \\
Diphthong & Dip & $\mathrm{I} @, \mathrm{U} @$, aI, aU, E@, eI, OI, @U \\
Silence & Sil & silence, short inter-word pause \\
\hline
\end{tabular}

\subsection{Training Data}

The training data for all systems was an ad-hoc corpus originally assembled for other purposes. The subjects were all speakers of Standard British English, and the utterances were a mixture of single words, phrases and complete sentences of varying lengths. The recordings were made with different equipment and at different sampling rates, digitally re-sampled to $16 \mathrm{kHz}$. The database consists of over 23,000 utterances, making a total of 48,000 spoken words taken from a vocabulary of 16,000 .

The phoneme strings to be aligned with the speech were based on a lexicon compiled from several sources, with manually edited transcriptions for any words in the database that were not in any of the lexica. For sentences and phrases, an optional 'short-pause' label was inserted between words. No post-lexical rules were applied, so some of the phonetic transcriptions may have been unrealistic.

\subsection{Alignment Systems}

All the alignment systems were based on Gaussian mixture Continuous-Density Hidden Markov Models (CD-HMMs), built with the HTK toolkit [6]. They were based on the same lexicon and were trained on the same data and with the same procedures and training parameters. The details which were changed between systems are as follows:

\subsubsection{Signal Analysis}

To ensure sufficient diversity in the alignments produced by the different systems, three different preprocessors were used to produce observation vectors: 
1. Mel-Frequency Cepstral Coefficients (MFCCs) [7] with a $20 \mathrm{~ms}$ time window for each frame, and one frame every $10 \mathrm{~ms}$.

2. Linear Prediction Cepstra (LP-Cepstra) [8] with a $24 \mathrm{~ms}$ time window, one frame every $8 \mathrm{~ms}$.

3. Auditory Description Vectors (ADVs) [9], with a frame rate of one every $10 \mathrm{~ms}$.

\subsubsection{HMM Structure}

The numbers of states and of Gaussian mixtures per state, were the same for all phoneme models within each system, but were varied between systems to produce different alignment results.

The number of states per phoneme were varied from 2 to 5 .

For 2 and 3 state models, a strict left-right topology was used with no skips. For 4 and 5 state models, 1 -state skips were allowed so that shorter phonemes could still be modelled without extending the states of one model into neighbouring phonemes.

The number of Gaussian mixtures per state was set to either 1, 2, 4, or 8 for all phoneme models in each system.

Thus for each of the 3 pre-processors, there are 4 choices of states per model, and 4 choices of mixtures per state, making a total of $3 \times 4 \times 4=48$ alignment systems.

\subsubsection{Training Procedure}

The training process used embedded re-estimation via the Baum-Welch algorithm [10], applied in three phases:

- Training from flat-start HMMs, initialised to the global means of all the training data, to produce single-mixture phoneme, silence, and short-pause models.

- Disambiguation of alternative pronunciations (including presence or absence of inter-word pauses) followed by retraining of the models.

- Disambiguation as before, and an increase in the number of mixtures in appropriate states (using a randomised duplication of each existing mixture), followed by final retraining of the full models.

Baum-Welch training was iterated four times at each stage.

\subsection{Comparisons}

The phonetic labels for each system were generated by conventional Viterbi alignment of the phonetic HMMs with the respective speech observation vectors. Each label from each alignment system was then compared with the equivalent label from each of the other systems. For each system-pair, the time offset between the labels was calculated. For the broad-class phoneme experiments, these were grouped according to the broad class of the phonemes involved. For brevity these groups will be referred to as "class-transitions" because they correspond to the transition from one broad phonetic class to another. The transitions in the other, ungrouped, experiments will be referred to as "phoneme-transitions".

For each combination of class / phoneme-transition and system-pair, histograms were constructed to show how frequently each time discrepancy was observed. The histogram bins were set to $10 \mathrm{~ms}$ width (the worst-case time resolution obtainable with the front ends used here). Some typical histograms are shown in Figures 1 to 3.

Figure 1 shows the class-transition distribution for a system-pair; One system labels the transitions $32 \mathrm{~ms}$ earlier than the other, but the variance is small, so the pair of systems are mutually consistent. Figure 2 shows the distribution for a transition which is labelled inconsistently by the two systems so the histogram is too broad. Finally, Figure 3 is a multi-moda distribution which, again, does not constitute a valid agreement between the systems.

In order to decide which class-transitions are reliable and which are not, the respective histogram is examined, and if more than $75 \%$ of the discrepancies fall in two adjacent bins, the two alignment systems are deemed to agree. This is quite a strict criterion - the systems must agree to within their characteristic time resolution on the vast majority of each transition.

Two bins are used in the calculation since the peak of the underlying continuous distribution might lie close to the edge of a bin, in which case the magnitude of the true peak would be divided between the two bins.

This histogram-based approach is used because it makes no strong assumptions about the distribution of values (which does not need to be Gaussian, continuous, or uni-modal), and even if one system of each pair consistently places its labels earlier or later than the other, they are not penalised. As long as the discrepancies are consistent, they will still be identified as agreeing with each other.
Finally, the overall consistency with which any given classtransition is identified is quantified by counting how many system-pairs agree. In these experiments there are 48 different systems so there are $(48 \times 47) / 2=1128$ possible pairs. The more pairs agree, the more reliable the labelling of that classtransition.

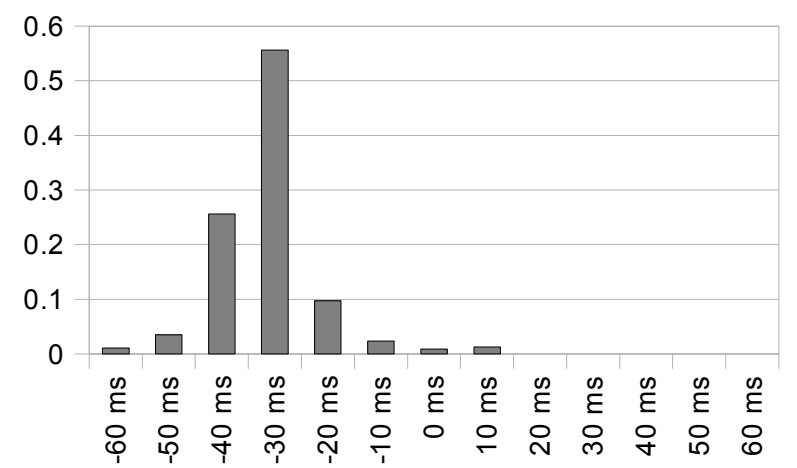

Figure 1: Normalised time discrepancy histogram for Sil $\rightarrow$ Nas transitions, comparing a system using ADVs for acoustic features with 2 states and 1 mixture versus one using LP Cepstra, with 3 states and 4 mixtures.

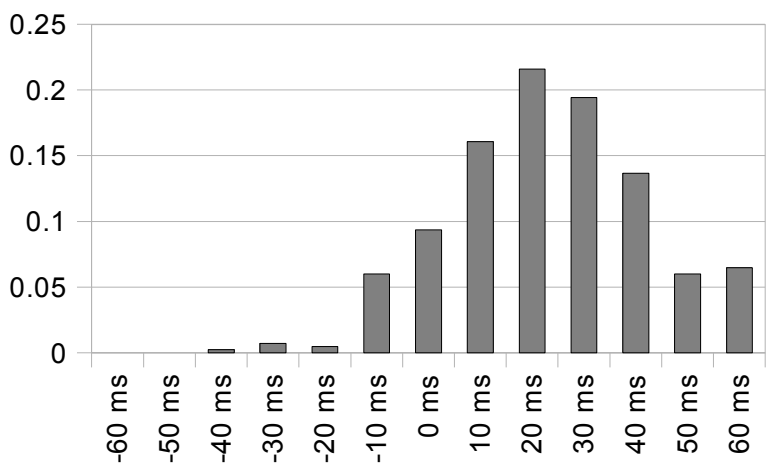

Figure 2: Normalised time discrepancy histogram for $\mathrm{App} \rightarrow$ Sil transitions, comparing ADVs, 2 states,

1 mixture versus LP Cepstra, 3 states, 8 mixtures

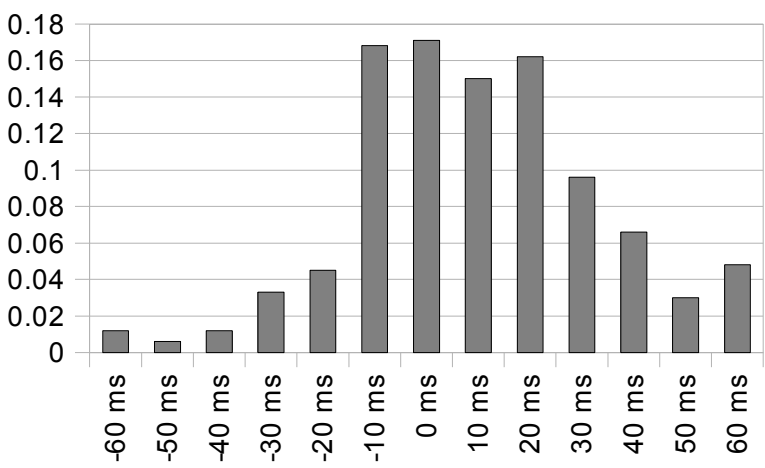

Figure 3: Normalised time discrepancy histogram for Dip $\rightarrow$ Sil transitions, comparing ADVs, 2 states, 1 mixture versus LP Cepstra, 2 states, 1 mixture

\section{Results}

The broad-class results are shown in Table 2, with the background colour denoting the magnitude (to make the structure in the figures more clear). The darker the cell, the more reliable the class-transition. There are some values missing from Table 2. There were no occurrences of an affricate-to-affricate transition in the data used for these experiments, and there were insufficient examples of affricateto-nasal transitions to calculate meaningful statistics. Of course a silence-to-silence transition is nonsensical! 
Table 2 Number of agreeing system-pairs for each class-transition

\begin{tabular}{|c|c|c|c|c|c|c|c|c|c|}
\hline \multirow{2}{*}{\multicolumn{2}{|c|}{$\begin{array}{c}\text { Transitions } \\
\text { Class } \mathrm{A} \rightarrow \text { Class B }\end{array}$}} & \multicolumn{8}{|c|}{ Class B } \\
\hline & & Plo & Aff & Frc & Nas & App & Vow & Dip & Sil \\
\hline \multirow{8}{*}{ Class A } & Plo & 19 & 39 & 70 & 84 & 166 & 441 & 469 & 57 \\
\hline & Aff & 369 & - & 25 & - & 63 & 688 & 753 & 123 \\
\hline & Frc & 438 & 86 & 22 & 456 & 133 & 423 & 537 & 116 \\
\hline & Nas & 241 & 632 & 247 & 5 & 52 & 332 & 396 & 84 \\
\hline & App & 208 & 156 & 251 & 60 & 5 & 26 & 9 & 103 \\
\hline & Vow & 332 & 365 & 254 & 182 & 8 & 1 & 1 & 78 \\
\hline & Dip & 361 & 437 & 260 & 170 & 6 & 0 & 4 & 77 \\
\hline & Sil & 923 & 395 & 299 & 817 & 713 & 513 & 516 & - \\
\hline
\end{tabular}

The corresponding table for the ungrouped phonemetransitions would be impractical to present here, but they have been compared to the grouped results and some of the more interesting observations are summarised below.

\subsubsection{Utterance-Initial Transitions}

On average, $72 \%$ of the system-pairs agree on silence-tophoneme transitions, but a significant number of silence-tophoneme transitions were identified identically by $99 \%$ or more of all system-pairs:

$$
\text { silence } \rightarrow / \mathrm{S} /, / \mathrm{d} /, / \mathrm{b} /, / \mathrm{w} /, / 1 /, / \mathrm{r} /, / \mathrm{m} /, / \mathrm{n} / \text {, or } / \mathrm{O} /
$$

Conversely, the least reliable silence-to-phoneme transitions (agreed upon by $40 \%$ or less of system-pairs) are:

$$
\text { silence } \rightarrow / \mathrm{v} /, / \mathrm{T} /, / \mathrm{h} /, / \mathrm{f} / \text {, or } / \mathrm{tS} /
$$

\subsubsection{Utterance-Final Transitions}

On average, only $13 \%$ of the system-pairs agree on phonemeto-silence transitions, and only the following transitions are consistently identified by more than $25 \%$ of system-pairs:

$$
\text { /s/ or } / \mathrm{S} / \rightarrow \text { silence }
$$

The following utterance-final transitions are the ones identified least accurately, with less than $5 \%$ of system pairs agreeing on their timings:

$$
/ \mathrm{k} / \text { or } / \mathrm{v} / \rightarrow \text { silence }
$$

\subsubsection{Most Reliable Transitions}

A number of phoneme-transitions are agreed upon by over $99 \%$ of the system-pairs. They are listed here:

$$
\begin{aligned}
/ \mathrm{s} / \rightarrow / @ \mathrm{U} /, / \mathrm{eI} /, / \mathrm{aI} /, / \mathrm{O} /, / \mathrm{E} /, / \mathrm{u} /, / \mathrm{i} /, / \mathrm{V} /, \text { or } / \mathrm{p} / \\
/ \mathrm{t} / \rightarrow / \mathrm{eI} /, / \mathrm{u} /, \text { or } / \mathrm{i} / \\
/ \mathrm{f} / \rightarrow / \mathrm{A} / \\
/ \mathrm{z} / \rightarrow / \mathrm{eI} /
\end{aligned}
$$

The vast majority of these correspond to a change in excitation from either a fricative or unvoiced stop to a voiced phoneme (vowel or diphthong).

\subsubsection{Most Unreliable Transitions}

The following phoneme-transitions are only consistent between $1 \%$ or less of the system-pairs:

$$
\begin{gathered}
/ \mathrm{U} @ /, / \mathrm{O} /, / \mathrm{E} /, / @ /, / \mathrm{u} /, \text { or } / \mathrm{i} / \rightarrow / \mathrm{l} / \\
/ \mathrm{a} / \mathrm{or} / \mathrm{aU} / \rightarrow / \mathrm{l} / \\
/ \mathrm{j} / \mathrm{u} / \\
/ \mathrm{U} @ / \mathrm{r} / \mathrm{r} / \mathrm{h} / \\
/ 1 / \rightarrow / \mathrm{eI} /, \text { or } / \mathrm{w} /
\end{gathered}
$$

$$
\begin{aligned}
& / \mathrm{s} / \rightarrow / \mathrm{s} / \\
& / \mathrm{t} / \rightarrow / \mathrm{t} /
\end{aligned}
$$

Most of these involve transitions either to or from diphthongs or approximants.

It should be borne in mind that the $/ \mathrm{s} / \rightarrow / \mathrm{s} /$ and $/ \mathrm{t} / \rightarrow / \mathrm{t} /$ transitions were most likely inconsistent simply because they were not articulated as transcribed. Most speakers will assimilate them into a single phoneme or insert a short pause between them, if trying to articulate clearly. This is a problem with the simple lexicon-based approach used both in this paper, and in most current speech recognition systems.

\section{Discussion}

An important feature of the analysis is that it allows systematic differences to be separated from unpredictable alignment errors. For instance, Figure 1 shows a pair of alignment systems that have a large systematic difference in where they put a phoneme boundary, but they disagree by a consistent amount. Such systematic disagreements can be as large as (or sometimes larger than) the unpredictable alignment errors. This reflects the fact that phoneme boundaries are ill-defined and that each alignment system embodies its own, slightly different definition of phonemes.

Looking at Table 2, the most reliable transitions are the ones from silence to the other phonetic classes. In particular, the most reliable of all are silence-to-plosive and silence-to-nasal. Conversely, the least reliable are among vowels, approximants and diphthongs. The transitions into silence are also relatively unreliable and variable. These broad-class conclusions are supported by results for individual phonemes.

The range of agreement scores is remarkably large: some phoneme-transitions (e.g. silence $\rightarrow / \mathrm{n} /$ ) are consistent between $99 \%$ of system-pairs, while some $($ e.g. $/ \mathrm{O} / \rightarrow / 1 /)$ are almost never consistent.

Table 3 summarises the ranges of reliability for different class-transitions. In terms of broad classes, those involving transitions between affricates and vowels, diphthongs, or nasals, seem to be more robust than most. As would be expected, many of the most precisely identifiable classtransitions correspond to transitions from one form of excitation to another - voiced, fricative, affricate, stop, etc. and most of the rest consist of abrupt changes to the vocal tract configuration - between nasals, vowels, and approximants, for example.

However, it also appears that in general, diphthongs produce more precisely identifiable labels than vowels. The most precisely identifiable boundaries of all are silence-toplosive.

These discrepancies observed between system-pairs are largely comparable with those between human segmenters, as documented by [1][3]. The pattern of these human errors is broadly similar to the those reported here, but with some notable discrepancies.

An inverse relationship would be expected between mean human segmentation error and the agreement scores presented here. This is supported for extreme pairs; for instance the classes with the three largest human errors (Nas $\rightarrow$ Nas, Vow $\rightarrow$ Vow, and Vow $\rightarrow$ App) have very low agreement scores in our work, and two classes with the smallest human errors (Frc $\rightarrow$ Plo and $\mathrm{Plo} \rightarrow$ Vow) are among our best-agreeing pairs. However, other pairs disagree. 
Table 3. Subjective transition groupings

\begin{tabular}{|c|l|}
\hline Agreeing Pairs & Class-Transitions \\
\hline $299-923$ & Silence $\rightarrow$ All \\
$600-800$ & Aff $\rightarrow$ Dip, Vow \\
& Nas $\rightarrow$ Aff \\
$400-600$ & Frc $\rightarrow$ Plo, Nas, Dip, Vow \\
& Plo $\rightarrow$ Dip, Vow \\
Dip $\rightarrow$ Aff \\
$300-400$ & Aff, Vow, Dip $\rightarrow$ Plo \\
& Nas $\rightarrow$ Vow, Dip \\
Vow $\rightarrow$ Aff \\
$200-300$ & App, Nas $\rightarrow$ Plo \\
& App, Nas, Dip, Vow $\rightarrow$ Frc \\
$100-200$ & App $\rightarrow$ Aff \\
& Frc, Plo $\rightarrow$ App \\
& Dip, Vow $\rightarrow$ Nas \\
& App, Dip, Vow $\rightarrow$ App, Dip, Vow \\
& App, Nas $\rightarrow$ App, Nas \\
& Plo $\rightarrow$ Plo, Nas \\
& Plo, Aff, Frc $\rightarrow$ Aff, Frc \\
& Aff $\rightarrow$ App \\
& All $\rightarrow$ Silence \\
\hline
\end{tabular}

Some of these differences may be attributed to the shortcomings of the lexicon-based approach used in this paper but this is an area which is the subject of further investigation.

\section{Conclusions}

While all phoneme boundaries are essentially artificial and, to a greater or lesser extent, arbitrary, some are more ambiguous than others. Consequently different boundaries should be given different weights when assessing the accuracy of any alignment or segmentation.

The results presented here have been derived without reference to any human alignments, but they broadly confirm independently assessed human labelling variations. It is possible that the more consistent boundaries are also easier for humans to decode, which might make them unusually important for human speech and language.

They indicate that the boundaries between vowels, diphthongs and approximants are highly ambiguous, and so do not provide an effective way of comparing alignment systems. Word endings (transitions from any phoneme to silence) are also relatively unreliable, and of little use in assessing accuracy.

On the other hand, word onsets (i.e. silence-to-phoneme transitions) are clearly identifiable almost regardless of the phoneme at the start of the word, and so are critical in assessing accuracy. Other reliable transitions include affricates-tovowels / diphthongs, and nasals-to-affricates. The most precisely identifiable class-transition of all is the silence-toplosive.

Ultimately these observations should not only affect the methods used to optimise alignment systems; they should also be taken into account when designing speech databases for the training of speech recognition and synthesis systems.

\section{Acknowledgement}

This project is supported by the Economic and Social Research Council (UK) via project RES-062-23-1172.

\section{References}

[1] Wesenick, M.-B., and Kipp, A., "Estimating the Quality of Phonetic Transcriptions and Segmentations of Speech Signals", Proceedings ICSLP-96, pages 129-132. IEEE, 1996.

[2] Vonwiller, J., Cleirigh, C., et al., "Development and Application of an Accurate and Flexible Automatic Aligner", International Journal of Speech Technology 1, pages $151-160,1997$.

[3] Cosi, P., Falavigna, D., and Omologo, M., "A Preliminary Statistical Evaluation of Manual and Automatic Segmentation Discrepancies", In EUROSPEECH-1991,
693-696.

http://www.isca-speech.org/archive/ eurospeech_1991/e91_0693.html (viewed 6/2009).

[4] Kominek, J., and Black, A., "A Family-of-Models Approach to HMM-Based Segmentation for Unit Selection Speech Synthesis", in Proc. Interspeech 2004, Jeju Island, Korea, 2004.

[5] Wells, J.C., "SAMPA computer readable phonetic alphabet". In Gibbon, D., Moore, R. and Winski, R. (eds.), 1997. Handbook of Standards and Resources for Spoken Language Systems. Berlin and New York: Mouton de Gruyter. Part IV, section B.

[6] Young, S., Evermann, G., Gales, M., Hain, T., Kershaw, D., Liu, X. (A.), Moore, G., Odell, J., Ollason, D., Povey, D., Valtchev, V., Woodland, P., The HTK Book (for HTK Version 3.4), Cambridge University Engineering Department 2006, Cambridge, UK.

[7] Bridle, J.S., and Brown, M.D., "An experimental automatic word recognition system," JSRU Report No. 1003, Joint Speech Research Unit, Ruislip, England. 1974.

[8] Atal, B. S., "Effectiveness of linear prediction characteristics of the speech wave for automatic speaker identification and verification", in J. Acoust. Soc. America, vol. 55, no. 6, pp.1304-1312, June, 1974.

[9] Kochanski, G., and Orphanidou, C., "Testing the ecological validity of repetitive speech". Proc. International Congress of Phonetic Sciences (ICPhS 2007), Saarbrücken, Germany. 10 Aug 2007.

[10] Baum, L. E., Petrie, T., Soules, G., and Weiss, N., "A maximization technique occurring in the statistical analysis of probabilistic functions of Markov chains", Ann. Math. Statist., vol. 41, no. 1, pp. 164-171, 1970. 